\title{
COMPLEX NETWORKS ANALYSIS BY SPECTRAL GRAPH THEORY
}

\author{
Nenad Jovanović1, \\ Zoran Jovanović², \\ Aleksandar Jevremović ${ }^{3}$ \\ ${ }^{1}$ Faculty of Technical Sciences - \\ University of Priština, \\ ${ }^{2}$ Business School of Applied Studies - \\ Blace, \\ ${ }^{3}$ Singidunum University - Belgrade
}

\begin{abstract}
: analysis of these networks. of analyzing algebraic connectivity in the network.

Keywords:

spectral graph, complex networks, algebraic connectivity.
\end{abstract}

Spectral graph theory is widely used in the process of characterization of complex networks properties, as well as the Internet infrastructure. This paper defines specific topological characteristics which can be used for the

Moreover, the paper describes the use of spectral graph theory in the process

\section{INTRODUCTION}

Specific topological graph characteristics are used for connectivity characterization and they have a significant impact on dynamic processes in complex networks, so that the analysis and synthesis of these networks is based on the use of metrics expressed by relevant topological characteristics.

Basic structural graph characteristics can be analyzed through considering graph topology. Graph topology determines how graph nodes are interconnected and what their mutual relations are.

Complex networks, as well as Internet infrastructure can be analyzed and modeled in terms of physical and logical topology. Logical topology includes collecting, measuring and analysis of parameters on IP level. Physical topology includes defining physical connection, which is one of the main research challenges [1].

Each node in the graph can be characterized by certain characteristics, Correspondence:

Nenad Jovanović

e-mail:

zoranjov2004@gmail.com such as the processing time, and each graph branch can be specified as a set of weighting functions, such as delay, bandwidth, packet loss...

Metrics are topological and can be calculated only by adjacency maon graph distance, connectivity and specter. trix. Topological metrics can be classified into metrics which are based 


\section{SPECTRAL GRAPH THEORY}

Spectral graph theory is based on its eigenvalues and eigenvectors. Eigenvalue of matrix $\mathrm{A}$ is a real number $\lambda$; it is the matrix equation:

$$
A x=\lambda x
$$

It has a nontrivial solution, which is called eigenvector. Eigenvalues of graph are eigenvalues of adjacency matrix. Graph specter $\mathrm{G}$ is determined by a set of its own eigenvalues of the adjacency matrix for the given graph.

Spectral graph theory uses specters of specific matrix, such as the adjacency matrix, the Laplacian matrix and normalized Laplacian matrix in order to obtain certain information about a graph.

For an unweighted, undirected graph G, the adjacency matrix is defined in the following way:

$$
A_{i, j}=\left\{\begin{array}{c}
1, \text { if there is a branch from i to } j \\
0, \text { other }
\end{array}\right.
$$

If a graph is weighted, then the adjacency matrix is defined as:

$$
A_{i, j}=\left\{\begin{array}{c}
w(i, j), \text { if there is a branch from i to } j \\
0, \text { other }
\end{array}\right.
$$

Eigenvalues of matrix $A$ are marked as $\lambda_{1}, \lambda_{2}, \ldots, \lambda_{n-1}, \lambda_{n}$ a and they represent matrix A specter.

Normalized adjacency matrix is determined:

$$
\hat{A}=\sqrt{D^{-1}} A \sqrt{D^{-1}}
$$

Degree matrix $\mathrm{D}$ is a diagonal matrix with node degree values of $d_{i}=1,2 \ldots n$ on the diagonal.

The Laplacian matrix is defined as:

$$
L=D-A
$$

Eigenvalues of matrix L are called the Laplacian eigenvalues and they are organized in a non-increasing order:

$$
\mu 1 \geq \mu 2 \geq \cdots \geq \mu_{n-1} \geq \mu_{n}
$$

A normalized Laplacian matrix is determined by:

$$
L_{N}=D^{-1 / 2} A D^{-1 / 2}
$$

The value of zeroes of the first eigenvalues of the Laplacian matrix indicates that network nodes are fully connected. Due to its characteristics, the Laplacian matrix specter is of greater significance, for most physical and chemical processes, than the adjacency matrix specter [2].

If graph $\mathrm{G}$ is not connected, the number of eigenvalues of the Laplacian matrix, which is equal to zero, is determined by the number of graph $\mathrm{G}$ components. For each graph $\mathrm{G}$ matrix, the specter is equal to the specter union of each graph component.

\section{TOPOLOGICAL METRICS}

Topological metrics, based on the spectral graph theory, are Algebraic connectivity, the Fiedler vector, Spectral radius, Principal eigenvector.

\section{Algebraic connectivity}

Eigenvalues of the Laplacian matrix are $\mu_{1} \geq \mu_{2} \geq$ $\ldots \geq \mu_{\mathrm{n}-1} \geq \mu_{\mathrm{n}}$.

The second lowest eigenvalue of the Laplacia matrix of graph $\mu_{\mathrm{n}-1}$ is called algebraic connectivity of graph G and it is a good parameter for measuring the graph connectivity. For example, the second lowest value is positive only if the graph is connected [3]. This value reflects the degree of connectivity of the total graph and it is used in the analysis of network robustness and synchronization.

\section{The Fiedler vector}

Eigenvector of the Laplacian matrix, which corresponds to the second lowest eigenvalue is called the Fiedler vector [3].

The Fiedler vector is used in the clustering process, which enables the graph to be split into two clusters, two node groups, so that the number of connections between clusters is minimized [4].

If $\mathrm{x}_{\mathrm{n}-1}$ is the Fiedler vector, then clustering is performed so that the nodes which correspond to positive values of vector $\mathrm{xn}-1$ are joined with the first cluster, and the nodes which correspond to the negative values of the vector $\mathrm{x}_{\mathrm{n}-1}$ are joined to the second cluster.

\section{Spectral radius}

If eigenvalues of the neighbouring matrix are organized in a decreasing order $\lambda_{1} \geq \lambda_{2} \geq \ldots, \lambda_{\mathrm{n}-1} \geq \lambda_{\mathrm{n}}$, the spectral radius $\rho$ is defined in the following way:

$$
\rho={ }_{1}^{\max }<\mathrm{i}<n \mid \lambda_{\mathrm{i}}
$$


The spectral radius of the graph has a significant role in the dynamic processes in the graph, such as spreading viruses in the network.

The Epidemic threshold is defined as $\tau_{c}=\frac{1}{\rho}$. When the effective infection rate in the network is $>\tau_{c}$, the network is infected, and if it is $\tau>\tau_{c}$ then there are no viruses in the network.

It can be concluded that the lower spectral radius corresponds to greater robustness in the network in terms of spreading viruses and greater protection from viruses can be achieved through minimization of the spectral radius [5].

\section{Principal eigenvector}

The Principal eigenvector of the neighbouring matrix $\mathrm{A}$ is a eigenvector which corresponds to the greatest eigenvalue, that is spectral radius $\rho$ [6].

The values of the principal eigenvector are directly connected to the relative significance of graph nodes (node centrality).

Google's PageRank algorithm uses the Principal eigenvector variation to mark the significance of a web page [7]. Coefficients of the Laplacian characteristic polynom and the greatest eigenvalues of the distance matrix are analyzed, as well as two invariants which are based on the graph specter - energy and the Estrada index.

\section{ANALYSIS - THE INFLUENCE OF THE SIGNAL STRENGTH IN A MESH NETWORK ON ALGEBRAIC CONNECTIVITY}

Mesh nodes are represented by graph nodes, and metrics values (for instance, OLSR parameters), which represent the signal strength among WMN nodes, are represented by graph branches (Fig. 1.).

Adjacency matrix, eigenvalues and eigenvectors of the Laplacian matrix for the mesh network in Fig. 1. are represented in Fig. 2.

We will continue to analyze how algebraic connectivity of networks changes by changing the signal strength in a network.

Table 1. shows the dependence of algebraic connectivity on the change of signal strength among various nodes for a constant value.

The value of algebraic connectivity for the graph from Fig. 1. is $\mu_{5}=66,339$. By changing the signal strength for the value of 5 , algebraic connectivity changed from
66,339 , when $\mathrm{L}_{1-5}$ changes from 12 to 17 , to 68,684 , when $\mathrm{L}_{2-5}$ changes from 10 to 15 or $\mathrm{L}_{1-2}$ changes from 10 to 15 .

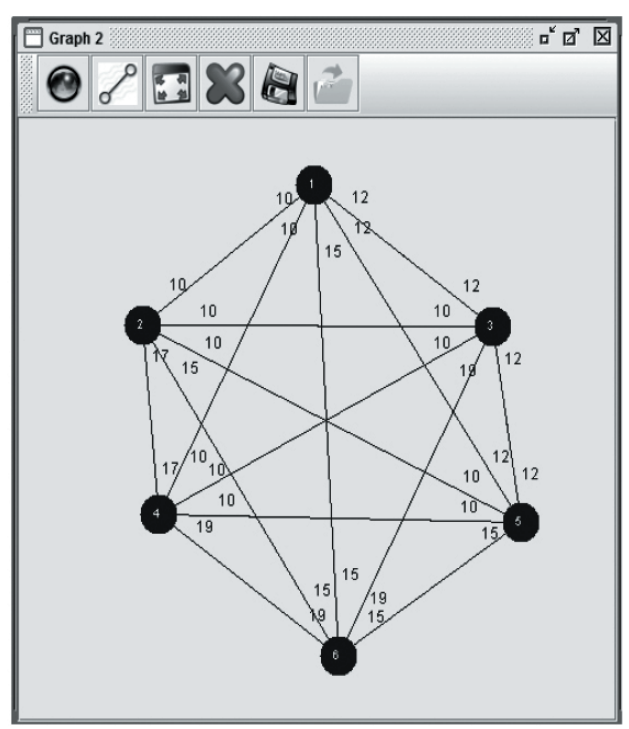

Fig. 1. Mesh network

\begin{tabular}{|c|c|c|c|c|c|}
\hline \multicolumn{4}{|c|}{ Adjacency matrix } & \multicolumn{2}{|c|}{$\square^{x} \square^{7}$ 区 } \\
\hline \multicolumn{6}{|c|}{ Graph } \\
\hline \multicolumn{3}{|c|}{ Adjacency matrix } & Eigenvalue & \multicolumn{2}{|c|}{ Eigenvector } \\
\hline 0 & 10 & 12 & 10 & 12 & 15 \\
\hline 10 & 0 & 10 & 17 & 10 & 15 \\
\hline 12 & 10 & 0 & 10 & 12 & 19 \\
\hline 10 & 17 & 10 & 0 & 10 & 19 \\
\hline 12 & 10 & 12 & 10 & 0 & 15 \\
\hline 15 & 15 & 19 & 19 & 15 & 0 \\
\hline
\end{tabular}

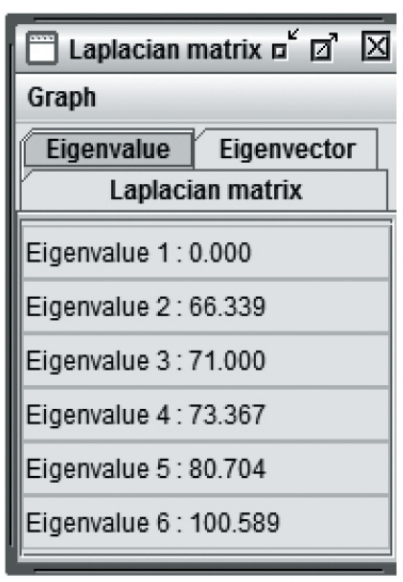




\begin{tabular}{|c|c|c|c|c|c|}
\hline \multicolumn{6}{|c|}{$\square$ Laplacian matrix } \\
\hline \multicolumn{6}{|c|}{ Graph } \\
\hline \multicolumn{3}{|c|}{ Laplacian matrix } & Eigenvalue & \multicolumn{2}{|c|}{ Eigenvector } \\
\hline 0.408 & -0.435 & -0.707 & 0.360 & 0.028 & 0.117 \\
\hline 0.408 & 0.598 & -0.000 & 0.182 & 0.660 & 0.082 \\
\hline 0.408 & -0.220 & 0.000 & -0.830 & 0.142 & 0.275 \\
\hline 0.408 & 0.462 & -0.000 & 0.054 & -0.724 & 0.304 \\
\hline 0.408 & -0.435 & 0.707 & 0.360 & 0.028 & 0.117 \\
\hline 0.408 & 0.031 & 0.000 & -0.126 & -0.135 & -0.894 \\
\hline
\end{tabular}

Fig. 2. Adjacency matrix, eigenvalues and eigenvectors of Laplacian matrix

The values of eigenvectors, which correspond to the algebraic connectivity are:

$-0.435,0.598,-0.220,0.462,-0,435$ i 0,031 .

Based on experimental results, it can be concluded:

1. Algebraic connectivity grows or remains the same if signal strength increases in the network.

2. Algebraic connectivity does not change if the signal strength changes among nodes which have the same values of the Fidler vector.

3. If the signal strength changes among nodes which have minimum and maximum values of the Fidler vector, algebraic connectivity grows to the maximum.

\begin{tabular}{ccccccc}
\hline & $\mathrm{L}_{1-2}$ & $\mathrm{~L}_{2-5}$ & $\mathrm{~L}_{1-4}$ & $\mathbf{L}_{4-5}$ & $\mathbf{L}_{2-3}$ & $\mathbf{L}_{3-4}$ \\
\hline $\boldsymbol{\mu}_{\mathrm{n}-1}$ & 68,684 & 68,684 & 68,167 & 68,167 & 67,966 & 67,530 \\
\hline
\end{tabular}

\begin{tabular}{ccccccc}
\hline & $\mathrm{L}_{2-6}$ & $\mathrm{~L}_{1-6}$ & $\mathrm{~L}_{5-6}$ & $\mathrm{~L}_{4-6}$ & $\mathrm{~L}_{3-6}$ & $\mathrm{~L}_{1-3}$ \\
\hline$\mu_{\mathrm{n}-1}$ & 67,442 & 66,895 & 66,895 & 67,021 & 66,537 & 66,429 \\
\hline
\end{tabular}

\begin{tabular}{cccc}
\hline & $\mathrm{L}_{3-5}$ & $\mathrm{~L}_{2-4}$ & $\mathrm{~L}_{1-5}$ \\
\hline$\mu_{\mathrm{n}-1}$ & 66,429 & 66,394 & 66,339 \\
\hline
\end{tabular}

Table 1 . Change of algebraic connectivity by changing signal strength

\section{CONCLUSION}

Understanding the evolution of complex networks is of crucial value for the analysis and modeling of networks and the Internet infrastructure.
This paper presents the use of the spectral graph theory in the process of complex network analysis. Topological metrics are defined, based on the spectral graph theory and the change of algebraic connectivity in a mesh network which is analyzed depending on the signal change in a network. A specific application has been developed which enables editing of a random graph of dependence and the calculation of all basic parameters of the spectral theory for the edited graph.

It has been demonstrated experimentally that algebraic connectivity is maximized when the signal strength increases among nodes which have minimum or maximum values of the Fidler vector, and algebraic connectivity does not change in case of changing the signal strength among nodes which have the same values as the Fidler vector.

\section{REFERENCES}

[1] Cetinkaya, E. K., Alenazi, M. J., Rohrer, J. P., \& Sterbenz, J. P. (2012, October). Topology connectivity analysis of internet infrastructure using graph spectra. In Ultra Modern Telecommunications and Control Systems and Workshops (ICUMT), 2012 4th International Congress on (pp. 752-758). IEEE.

[2] Mohar, B., Alavi, Y., Chartrand, G., \& Oellermann, O. R. (1991). The Laplacian spectrum of graphs. Graph theory, combinatorics, and applications, 2, 871-898.

[3] Fiedler, M. (1973). Algebraic connectivity of graphs. Czechoslovak mathematical journal, 23(2), 298-305.

[4] Verma, D., \& Meila, M. (2003). A comparison of spectral clustering algorithms. University of Washington Tech Rep UWCSE030501, 1, 1-18.

[5] Van Mieghem, P., Stevanović, D., Kuipers, F., Li, C., Van De Bovenkamp, R., Liu, D., \& Wang, H. (2011). Decreasing the spectral radius of a graph by link removals. Physical Review E, 84(1), 016101.

[6] Cioaba, S. M., \& Gregory, D. A. (2007). Principal eigenvectors of irregular graphs. Electron. J. Linear Algebra, 16, 366-379.

[7] Langville, A. N., \& Meyer, C. D. (2011). Google's PageRank and beyond: The science of search engine rankings. Princeton University Press. 